\title{
Electrochemical Dynamics of a Single Platinum Nanoparticle Collision Event for the Hydrogen Evolution Reaction
}

\author{
Zhi-peng Xiang ${ }^{+}$, Hai-qiang Deng ${ }^{+}$Pekka Peljo, Zhi-yong Fu, Su-li Wang, Daniel Mandler, \\ Gong-quan Sun,* and Zhen-xing Liang*
}

\begin{abstract}
Chronoamperometry was used to study the dynamics of Pt nanoparticle (NP) collision with an inert ultramicroelectrode via electrocatalytic amplification (ECA) in the hydrogen evolution reaction. ECA and dynamic light scattering $(D L S)$ results reveal that the NP colloid remains stable only at low proton concentrations $(1.0 \mathrm{mM})$ under a helium (He) atmosphere, ensuring that the collision events occur at genuinely single NP level. Amperometry of single NP collisions under a He atmosphere shows that each discrete current profile of the collision event evolves from spike to staircase at more negative potentials, while a staircase response is observed at all of the applied potentials under hydrogen-containing atmospheres. The particle size distribution estimated from the diffusion-controlled current in He agrees well with electron microscopy and DLS observations. These results shed light on the interfacial dynamics of the single nanoparticle collision electrochemistry.
\end{abstract}

$N_{\text {anoparticles (NPs) make a great impact in many appli- }}$ cations, such as energy, ${ }^{[1]}$ catalysis (for example, photocatalysis and electrocatalysis) ${ }^{[2]}$ sensors, ${ }^{[3]}$ electrochemiluminescence ${ }^{[4]}$ and spectroscopy, ${ }^{[5]}$ which is due to their fascinating physical and chemical properties. NP properties are mainly dependent on their composition, size, and shape. ${ }^{[6]}$ Hence, many studies have been conducted to elucidate the structureactivity relationships of NPs. ${ }^{[7]}$ Traditional ensemble measurements are limited to provide only averaged characteristics of

[*] Z.-P. Xiang, ${ }^{[+]}$Prof. Dr. Z.-Y. Fu, Prof. Dr. Z.-X. Liang Key Laboratory on Fuel Cell Technology of Guangdong Province, School of Chemistry and Chemical Engineering, South China University of Technology Guangzhou 510641 (P. R. China)

E-mail: zliang@scut.edu.cn

Dr. H.-Q. Deng, ${ }^{[+]}$Prof. Dr. D. Mandler

Institute of Chemistry, The Hebrew University of Jerusalem Jerusalem 9190401 (Israel)

Dr. P. Peljo

Laboratoire d'Electrochimie Physique et Analytique

École Polytechnique Fédérale de Lausanne (EPFL Valais Wallis) Rue de l'Industrie, 17, 1951 Sion (Switzerland)

Prof. Dr. S.-L. Wang, Prof. Dr. G.-Q. Sun

Fuel Cell \& Battery Division, Dalian National Laboratory for Clean Energy, Dalian Institute of Chemical Physics

Chinese Academy of Sciences, Dalian 116023 (P. R. China)

E-mail: gqsun@dicp.ac.cn

$\left.{ }^{+}\right]$These authors contributed equally to this work.

(-) Supporting information and the ORCID identification number(s) for

(DD the author(s) of this article can be found under:

https://doi.org/10.1002/anie.201712454. a large number of NPs with distribution of sizes and shapes in a typical sample. It is thus highly desirable to develop techniques to characterize structure-activity relationship of a single NP as a means of understanding and further tuning NP performance. Until now, spectroscopy (for example, single-molecule fluorescence microscopy, ${ }^{[8]}$ infrared nanospectroscopy ${ }^{[9]}$ local surface plasmon resonance, ${ }^{[10]}$ and tipenhanced Raman spectroscopy ${ }^{[11]}$ ) has become the mainstream method to study single molecules and nanoparticles. Nevertheless, spectroscopic methods are generally limited by either chemical selectivity (for example, limited by particular molecules) or spatial resolution (for example, diffraction limit). Electrochemical methods ${ }^{[12]}$ are emerging as an indispensable alternative and/or a complement to study single NPs either by immobilizing a NP onto a substrate electrode combined with scanning electrochemical microscopy $(\mathrm{SECM})^{[13]}$ or scanning electrochemical cell microscopy $(\mathrm{SECCM})^{[14]}$ or by stochastic collision/impact of individual NPs onto an ultramicroelectrode (UME) without a tricky immobilization step. ${ }^{[12]}$ The second electrochemical method can directly characterize NPs in the colloidal solution. The current signal induced by a NP collision can be either from bulk electrolysis $(\mathrm{BE})$ of the redox-active NP itself ${ }^{[15]}$ or from electrocatalytic amplification (ECA) when a NP catalyzes an inner-sphere-electron-transfer (ISET) reaction that is kinetically sluggish on the underlying UME ${ }^{[16]}$ ECA is superior to $\mathrm{BE}$ in terms of the detection sensitivity and temporal resolution.

The hydrogen evolution reaction (HER) is the simplest heterogeneous ISET reaction between (solvated) protons and underlying electrified electrodes. ${ }^{[17]}$ Accordingly, HER has served as a model reaction in single Pt NP collision detection by ECA. ${ }^{[16 a, b, 18]}$ The colloidal stability of Pt NPs and the explanation of the obtained electrochemical signal from HER on single Pt NPs are still elusive. ${ }^{[19]}$ The difficulty in interpreting the current profile in the ECA approach is due to the variation of the interactions between a NP and the substrate UME, resulting in either spikes or staircases. In the case where a NP sticks onto the UME biased at the diffusionlimited potentials, a current staircase is expected if no deactivation/poisoning of the NP catalyst occurs, ${ }^{[20]}$ while the catalyst deactivation can lead to a spiky response. Herein, we extensively investigate the mechanism underpinning the evolution of the current transient profile of HER on single Pt NPs from spike to staircase with varying electrode potentials under different gas atmospheres in the absence of oxygen (Scheme 1). It is found that the interfacial dynamics plays a key role in determining the current shape. Specifically, it is the drift in equilibrium potential and slower HER kinetics 


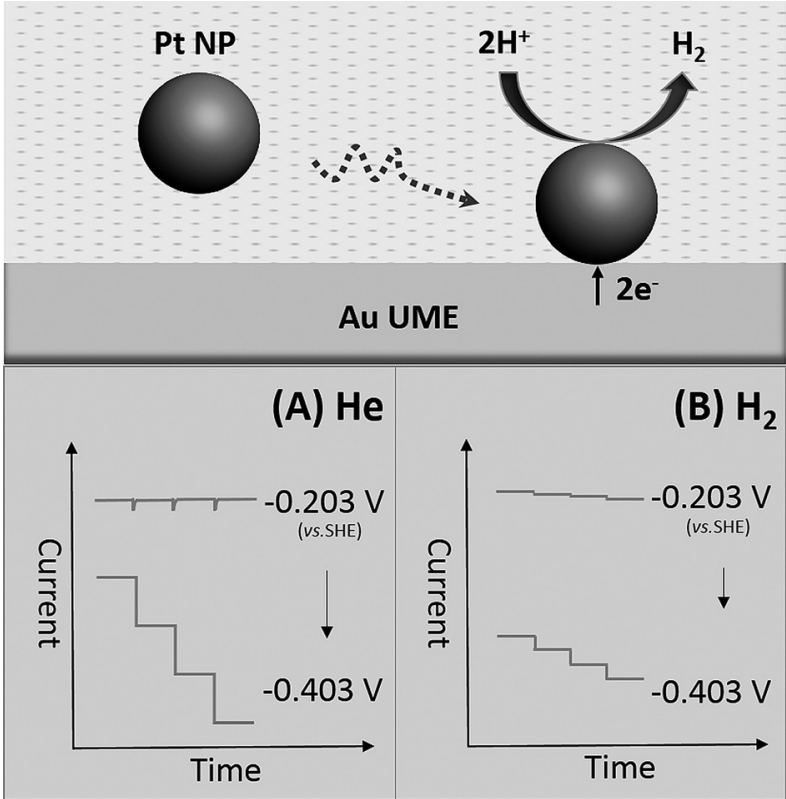

Scheme 1. Current profile upon a single Pt NP collision on a gold ultramicroelectrode (UME) under either a helium or hydrogen atmosphere at varied applied potentials.

compared to mass-transfer of protons ${ }^{[21]}$ in aqueous media to a nanometer-sized Pt surface ${ }^{[16 e, 22]}$ that result in a spiky current transient in helium-saturated solution at lower overpotentials. Nevertheless, a staircase-shaped current profile can be seen either in helium-saturated solution at higher overpotentials or in a hydrogen-containing solution even at lower overpotentials. The current shape is rationalized by the analysis of thermodynamics, kinetics, and finite-element simulations of HER. Our findings presented herein pave the way for electrocatalytic studies at a truly single nanoparticle level. ${ }^{[23]}$

First, the issue of NP colloidal instability was addressed to guarantee single NP electrochemical detection. The Pt NPs have a modal size of $71 \mathrm{~nm}$ in diameter (scanning electron microscopy, SEM) and a corresponding hydrodynamic diameter of $85 \mathrm{~nm}$ (dynamic light scattering, DLS; Supporting Information, Figure S1 and Table S1). Uniform current transients are observed in $\mathrm{He}$-saturated $1.0 \mathrm{~mm} \mathrm{HClO}_{4}$ solution (black trace in the Supporting Information, Figure S3A) at an applied overpotential of $0.10 \mathrm{~V}$ (Supporting Information, Table S3), indicating that individual nanoparticles rather than aggregates collide with the UME surface. In comparison, inhomogeneous current magnitudes, irregular time intervals, and lower impact frequencies of the ECA signals are observed in both 5.0 and $25 \mathrm{~mm} \mathrm{HClO}_{4}$ solutions (red and blue traces in the Supporting Information, Figure S3A) at the same applied overpotential, indicating that NPs are unstable and aggregate in the colloidal dispersion. It implies that proton concentration dictates the colloidal stability via electrostatic interactions between the double layer of the $\mathrm{NP}$ and $\mathrm{H}^{+}$. This observation was corroborated by DLS kinetic measurements under both ambient and $\mathrm{He}$ atmospheres, as shown in the Supporting Information, Figures S3B and S5, respectively. The experimental NP/UME collision frequency of $0.05 \mathrm{~Hz}$ (Supporting Information, Figure S4) in He-saturated $1.0 \mathrm{~mm} \mathrm{HClO}_{4}$ is in reasonable agreement with the calculated one of $0.09 \mathrm{~Hz}$ (Supporting Information, Equation S5). This deviation might originate from the experimental errors, ineffective collision events, ${ }^{[24]}$ Fermi-level equilibration of NP in close contact with the electrode, ${ }^{[25]}$ and the near-wall hindered diffusion ${ }^{[20 b, 26]}$ (more details can be seen in the Supporting Information, pages S9,S10). We employed $1.0 \mathrm{~mm} \mathrm{HClO}_{4}$ to carry out the ECA collision experiments throughout the work shown herein, although $\mathrm{Pt}$

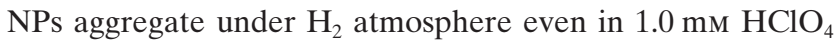
(blue trace in the Supporting Information, Figure S5).

ECA of HER observed upon NP collision should show a staircase current profile when NPs colliding with the electrode stick to the surface at sufficient overpotentials where the diffusion-limited regime of proton is reached. Instead, experimental results show a spiky profile (Supporting Information, Figure S3A). Effects of hydrogen-under-potential-deposition $\left(\mathrm{H}_{\mathrm{UPD}}\right)$, capacitive currents, or reduction of either oxygen or platinum oxide were excluded as the origin of the current spikes (Supporting Information, Figures S6,S7). Furthermore, the elastic collision of the NP may account for the spiky profile; however, the cyclic voltammograms (CVs) (Supporting Information, Figure S8) indicate that the collided Pt NPs stick onto the Au UME after their landings, rather than elastically collide and leave the electrode after impacting the UME.

It was suggested by Stevenson et al. ${ }^{[18]}$ that ECA reaction, working electrode, and electrolyte solution could affect the current transient shape. Herein, we found that the electrode potential also has a substantial effect on the current transient shape of a collision event. Figure $1 \mathrm{~A}$ shows the amperometric curves of $0.58 \mathrm{pm}$ Pt NPs colliding on a $25 \mu \mathrm{m}$ Au UME in Hesaturated $1.0 \mathrm{~mm} \mathrm{HClO}_{4}$. The current transient evolves from spike to staircase eventually while polarizing the electrode to more negative potentials. One possible explanation would be that the interfacial dynamics dictates the current transient shape: 1) drift in the equilibrium potential, and 2) slower HER kinetics compared to mass-transfer rate of $\mathrm{H}^{+}$to nanometer-sized Pt surface. ${ }^{[16 e, 22]}$

To gain further information, amperometric $i-t$ curves were collected under the same conditions except using $\mathrm{H}_{2}$-saturated solution (Figure $1 \mathrm{~B}$ ) as well as $\mathrm{He}-\mathrm{H}_{2}$ mixture with different partial pressures of $\mathrm{H}_{2}$ (Supporting Information, Figure S10). In all the cases, the current signal shows a staircase for each collision event independent of the applied potential. Figure $2 \mathrm{~A}$ shows the average current of the collision events as a function of the applied potentials plotted from Figure 1. The current first increases with decreasing the potential, and then levels off at more negative potentials $\leq$ $-0.293 \mathrm{~V}$ in helium-saturated solution (trace "a" in Figure $2 \mathrm{~A}$ ). At less negative potentials, the HER is at least partially controlled by kinetics; therefore, the current gradually increases with biasing the UME to more negative potentials. When the potential is sufficiently negative, the HER changes from the kinetics control to the diffusion control, which explains the subsequent presence of a current plateau. It is also interesting to note that the current magnitudes remain the same despite the considerable 

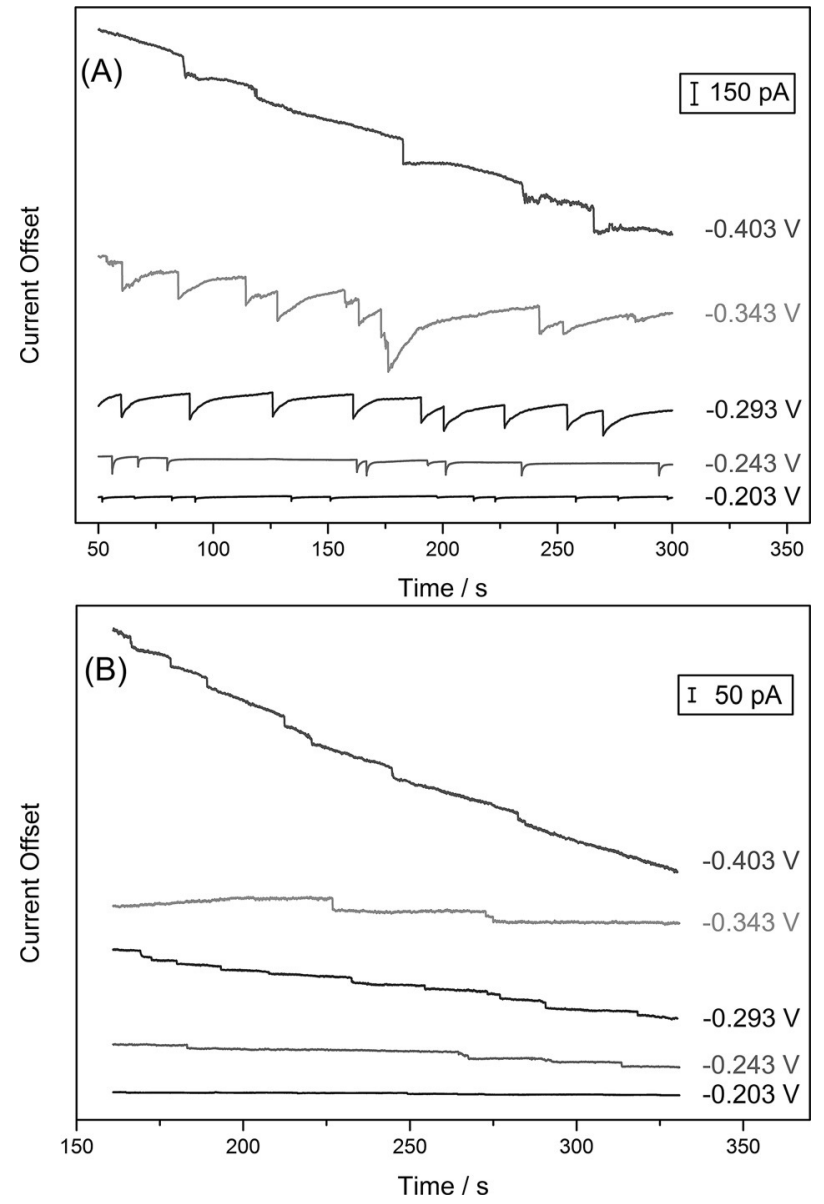

Figure 1. Amperometric $i-t$ curves of $0.58 \mathrm{pM}$ Pt NPs colliding with a $25 \mu \mathrm{m}$ Au UME biased from -0.203 to $-0.403 \mathrm{~V}$ vs. SHE. A) Hesaturated $1.0 \mathrm{mM} \mathrm{HClO}$ solution, and $\mathrm{B}) \mathrm{H}_{2}$-saturated $1.0 \mathrm{mM} \mathrm{HClO}_{4}$ solution.

change in the partial pressure of hydrogen from 0.2 to $1 \mathrm{~atm}$ (Figure 2B). However, the current transient magnitude in a $\mathrm{He}$ atmosphere is higher than that in $\mathrm{H}_{2}$ containing atmospheres, which is unexpected as the current should depend only on the diffusion of protons. The blocking effect of hydrogen bubble formation on the NP surface ${ }^{[27]}$ was also excluded as a possible origin for the spike response under a He atmosphere (see the Supporting Information, page S18, for further details).

To further address this concern, the HER was simulated with finite element method using COMSOL Multiphysics (see the Supporting Information, pages S24, S25 for more details), assuming the Volmer-Tafel mechanism $\left(\mathrm{H}^{+}+\mathrm{e}^{-}+\right.$ $\mathrm{Pt} \leftrightarrow \mathrm{H}-\mathrm{Pt}$ and $\left.2 \mathrm{H}-\mathrm{Pt} \leftrightarrow \mathrm{H}_{2}+2 \mathrm{Pt}\right)$ and with the parameters reported by Compton et al., ${ }^{[28]}$ with Pt NP radius of $35 \mathrm{~nm}$ and $0.63 \mathrm{~mm}$ of protons in the bulk solution. The initial bulk hydrogen concentration, which is experimentally controlled by the partial pressure in the mixture, used in simulation varies from 0 to $0.79 \mathrm{~mm}^{\left[{ }^{[28 a]}\right.}$ The simulation results are tabulated in the Supporting Information, Table S5. The currents obtained from the simulation (trace "c" in Figure $2 \mathrm{~A}$ ) fit well with the experimental data in He-saturated solutions. In comparison, Figure 2 reveals that the current
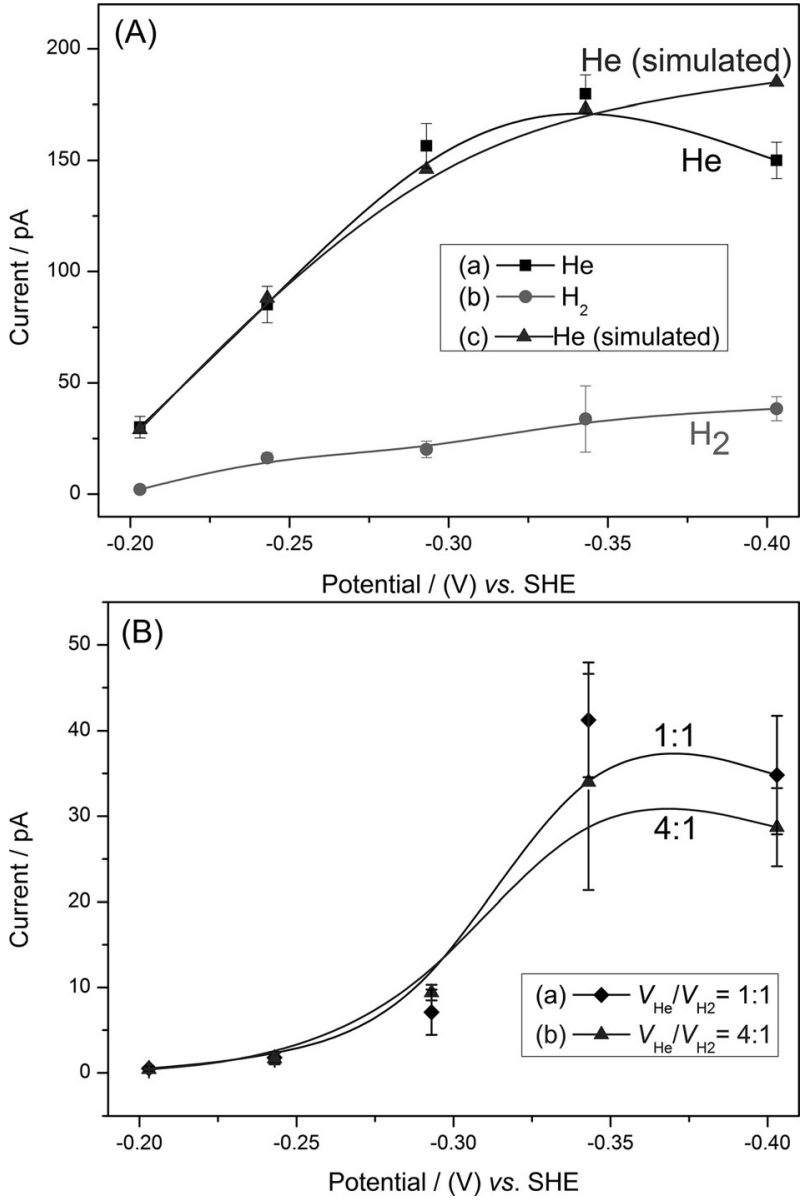

Figure 2. A) The average current transient magnitudes of the collision events from Figure 1 as a function of applied potentials in a) heliumsaturated $1.0 \mathrm{~mm} \mathrm{HClO}_{4}$ solution, b) hydrogen-saturated $1.0 \mathrm{~mm}$ $\mathrm{HClO}_{4}$ solution, and c) from simulation under $100 \%$ helium atmosphere. B) The average current magnitudes of the collision events (Supporting Information, Figure S10) biased from -0.203 to $-0.403 \mathrm{~V}$ in $1.0 \mathrm{mM} \mathrm{HClO}_{4}$ solution saturated with a $\mathrm{He}-\mathrm{H}_{2}$ mixture.

response under $\mathrm{H}_{2}$-containing atmospheres is always at least five times lower (see for example the experimental currents at $-0.343 \mathrm{~V}$ in Figure 2 A).

A possible explanation is that the dissolved hydrogen molecule spontaneously undergoes chemical dissociation into $\mathrm{H}$ atoms and adsorb/absorb onto/inside the Pt NPs. As a consequence, the HER electrocatalysis can be passivated by the hydrogen absorption into the crystal lattice of Pt NPs, ${ }^{[29]}$ as suggested earlier by Bard et al. ${ }^{[16 a, b]}$ In this process, the change in the Gibbs energy is sufficiently negative such that the process can proceed completely, and is independent of the partial pressure of hydrogen. It is inferred that the Pt NPs will become passivated to a similar degree even though the partial pressure of hydrogen remarkably varies from 1 to 0.2 atm. As such, the current signal of NP collision is essentially the same in hydrogen-containing solution considering the same proton concentration. Interestingly, Compton and co-workers observed similar passivation effect for the hydrogen oxidation reaction (HOR) on single Pt NPs. ${ }^{[28 b]}$ Furthermore, the instability of the Pt NPs colloid revealed by DLS (Supporting Information, Figure S5) can be seen as a collateral evidence 
for the hydrogen effect. In such solutions, the Fermi level of the Pt NP is equilibrated with the $\mathrm{H}^{+} / \mathrm{H}_{2}$ redox couple. ${ }^{[30]} \mathrm{At}$ these potentials, the citrate may be replaced by the dissociated $\mathrm{H}$ atoms on the NP surface, thus degrading the colloidal stability.

Furthermore, the NP size distribution is calculated by using the steady-state current under $100 \%$ helium atmosphere (Figure 1 A) and the Supporting Information, Equation S13. The modal size is found to be ca. $67 \mathrm{~nm}$ (Supporting Information, Figure S11), which coincides well with the SEM result of about $71 \mathrm{~nm}$ (Supporting Information, Figure S1 and Table S1).

In summary, we investigated the effect of the interfacial dynamics on the current transient profile of a single Pt NP collision via the ECA towards the HER. First, Pt NP aggregation as a function of proton concentration was studied to achieve a genuinely single NP collision. It is found that the $70 \mathrm{~nm}$ diameter Pt NPs remain stable against aggregation for a long duration $(\geq 30 \mathrm{~min})$ at proton concentrations of $\leq 1.0 \mathrm{~mm}$. Afterwards, the chronoamperograms were collected to track single NP collisions at different electrode potentials under varied atmospheres. It is found that the current transient evolves from spike to staircase upon applying more negative potentials in He-saturated solution. Thermodynamics analysis indicates that the shape of the chronoamperogram depends on the interfacial dynamics, which results in a drift in equilibrium potential and slower HER kinetics than mass transfer under $\mathrm{He}$ atmosphere at insufficient overpotentials. In comparison, the current signal is staircase-like at all investigated potentials in $\mathrm{H}_{2}$-containing solution, but at least five times lower in magnitude than expected. This difference is supposed to originate from the passivation of the HER electrocatalyst by the hydrogen absorption into the crystal lattice of Pt NPs, ${ }^{[29]}$ as suggested earlier by Bard et al. ${ }^{[16 a, b]}$ The calculated Pt NP size distribution via the electrochemical method in He-saturated solution correlates well with both the DLS and the SEM results. Our work presented herein sheds light on the nanoelectrode electrochemistry via ECA, which is likely to be a cornerstone to study electrochemistry at a genuinely single nanoparticle level.

\section{Acknowledgements}

This work was jointly supported by the National Natural Science Foundation of China (Nos. 21676106, 21476087), the Science and Technology Program of Guangzhou (201704030065), and the Science and Technology Program of Guangdong (2017A050506015). D.M. acknowledges the support by the Israeli Ministry of Science and Technology (Contract No. 3-12610). H.D. acknowledges the financial support from the PBC Fellowship jointly sponsored by the Planning and Budgeting Committee of the Council for Higher Education in Israel and the Hebrew University of Jerusalem and also appreciates Prof. Daniel Mandler for hosting his postdoctoral studies. P.P. acknowledges the financial support from the Swiss National Science Foundation, grant Ambizione Energy 160553.

\section{Conflict of interest}

The authors declare no conflict of interest.

Keywords: collisions - electrocatalytic amplification . hydrogen evolution reaction - interfacial dynamics . nanoparticles

How to cite: Angew. Chem. Int. Ed. 2018, 57, 3464-3468 Angew. Chem. 2018, 130, 3522-3526

[1] M. K. Debe, Nature 2012, 486, 43-51.

[2] Y. Xia, H. Yang, C. T. Campbell, Acc. Chem. Res. 2013, 46, 1671 1672.

[3] X. Zhong, L. Qiao, N. Gasilova, B. Liu, H. H. Girault, Anal. Chem. 2016, 88, 6184-6189.

[4] M. Hesari, Z. Ding, Acc. Chem. Res. 2017, 50, 218-230.

[5] J. F. Li, Y. F. Huang, Y. Ding, Z. L. Yang, S. B. Li, X. S. Zhou, F. R. Fan, W. Zhang, Z. Y. Zhou, D. Y. Wu, B. Ren, Z. L. Wang, Z. Q. Tian, Nature 2010, 464, 392-395.

[6] a) A. Henglein, Chem. Rev. 1989, 89, 1861-1873; b) G. Schmid, Chem. Rev. 1992, 92, 1709-1727; c) G. F. Long, X. H. Li, K. Wan, Z. X. Liang, J. H. Piao, P. Tsiakaras, Appl. Catal. B 2017, 203, 541-548.

[7] K. Wan, Z. P. Yu, X. H. Li, M. Y. Liu, G. Yang, J. H. Piao, Z. X. Liang, ACS Catal. 2015, 5, 4325-4332.

[8] a) W. E. Moerner, L. Kador, Phys. Rev. Lett. 1989, 62, 2535 2538; b) J. B. Sambur, P. Chen, Annu. Rev. Phys. Chem. 2014, 65, $395-422$.

[9] C. Y. Wu, W. J. Wolf, Y. Levartovsky, H. A. Bechtel, M. C. Martin, F. D. Toste, E. Gross, Nature 2017, 541, $511-515$.

[10] X. Shan, I. Díez-Pérez, L. Wang, P. Wiktor, Y. Gu, L. Zhang, W. Wang, J. Lu, S. Wang, Q. Gong, J. Li, N. Tao, Nat. Nanotechnol. 2012, 7, 668-672.

[11] J. H. Zhong, X. Jin, L. Y. Meng, X. Wang, H. S. Su, Z. L. Yang, C. T. Williams, B. Ren, Nat. Nanotechnol. 2017, 12, 132-136.

[12] a) W. Cheng, R. G. Compton, TrAC Trends Anal. Chem. 2014, 58, 79-89; b) A. Bard, A. Boika, S. Kwon, J. Park, S. Thorgaard, Nanoelectrochemistry, CRC, Boca Raton, FL, 2015, pp. $241-$ 292; c) M. D. Scanlon, P. Peljo, M. A. Mendez, E. Smirnov, H. H. Girault, Chem. Sci. 2015, 6, 2705-2720; d) T. J. Anderson, B. Zhang, Acc. Chem. Res. 2016, 49, 2625-2631.

[13] a) T. Sun, Y. Yu, B. J. Zacher, M. V. Mirkin, Angew. Chem. Int. Ed. 2014, 53, 14120-14123; Angew. Chem. 2014, 126, 1434414347; b) Y. Yu, T. Sun, M. V. Mirkin, Anal. Chem. 2015, 87, $7446-7453$.

[14] A. G. Güell, K. E. Meadows, P. V. Dudin, N. Ebejer, J. V. Macpherson, P. R. Unwin, Nano Lett. 2014, 14, 220-224.

[15] a) Y. G. Zhou, N. V. Rees, R. G. Compton, Angew. Chem. Int. Ed. 2011, 50, 4219-4221; Angew. Chem. 2011, 123, 4305-4307; b) H. S. Toh, R. G. Compton, Chem. Sci. 2015, 6, 5053-5058; c) H. Deng, J. E. Dick, S. Kummer, U. Kragl, S. H. Strauss, A. J. Bard, Anal. Chem. 2016, 88, 7754-7761; d) A. Feng, W. Cheng, R. G. Compton, Chem. Sci. 2016, 7, 6458-6462; e) K. Shimizu, K. Tschulik, R. G. Compton, Chem. Sci. 2016, 7, 1408-1414.

[16] a) X. Xiao, A. J. Bard, J. Am. Chem. Soc. 2007, 129, 9610-9612; b) X. Xiao, F. R. F. Fan, J. Zhou, A. J. Bard, J. Am. Chem. Soc. 2008, 130, 16669-16677; c) S. E. F. Kleijn, S. C. S. Lai, T. S. Miller, A. I. Yanson, M. T. M. Koper, P. R. Unwin, J. Am. Chem. Soc. 2012, 134, 18558-18561; d) R. Dasari, D. A. Robinson, K. J. Stevenson, J. Am. Chem. Soc. 2013, 135, 570-573; e) Z. Guo, S. J. Percival, B. Zhang, J. Am. Chem. Soc. 2014, 136, 8879-8882; f) X. Huang, H. Deng, C. Liu, J. Jiang, Q. Zeng, L. Wang, Chem. Eur. J. 2016, 22, 9523-9527.

[17] A. J. Bard, L. R. Faulkner, Electrochemical Methods, 2nd ed., Wiley, New York, 2001. 
[18] R. Dasari, B. Walther, D. A. Robinson, K. J. Stevenson, Langmuir 2013, 29, 15100-15106.

[19] D. A. Robinson, A. M. Kondajji, A. D. Castañeda, R. Dasari, R. M. Crooks, K. J. Stevenson, J. Phys. Chem. Lett. 2016, 7, $2512-2517$.

[20] a) S. J. Kwon, H. Zhou, F. R. F. Fan, V. Vorobyev, B. Zhang, A. J. Bard, Phys. Chem. Chem. Phys. 2011, 13, 5394-5402; b) E. Kätelhön, R. G. Compton, Chem. Sci. 2014, 5, 4592-4598.

[21] a) N. Agmon, Chem. Phys. Lett. 1995, 244, 456-462; b) O Markovitch, H. Chen, S. Izvekov, F. Paesani, G. A. Voth, N. Agmon, J. Phys. Chem. B 2008, 112, $9456-9466$.

[22] a) R. M. Penner, M. J. Heben, T. L. Longin, N. S. Lewis, Science 1990, 250,1118-1121; b) S. J. Percival, B. Zhang, J. Phys. Chem. C 2016, 120, 20536-20546.

[23] S. E. F. Kleijn, S. C. S. Lai, M. T. M. Koper, P. R. Unwin, Angew. Chem. Int. Ed. 2014, 53, 3558-3586; Angew. Chem. 2014, 126 , $3630-3660$.

[24] V. Brasiliense, A. N. Patel, A. Martinez-Marrades, J. Shi, Y. Chen, C. Combellas, G. Tessier, F. Kanoufi, J. Am. Chem. Soc. 2016, $138,3478-3483$.
[25] P. Peljo, J. A. Manzanares, H. H. Girault, Chem. Sci. 2017, 8, $4795-4803$.

[26] a) S. Eloul, R. G. Compton, J. Phys. Chem. Lett. 2016, 7, 43174321; b) S. V. Sokolov, S. Eloul, E. Katelhon, C. BatchelorMcAuley, R. G. Compton, Phys. Chem. Chem. Phys. 2017, 19, $28-43$.

[27] L. Luo, H. S. White, Langmuir 2013, 29, 11169-11175.

[28] a) X. Jiao, C. Batchelor-McAuley, E. Kätelhön, J. Ellison, K. Tschulik, R. G. Compton, J. Phys. Chem. C 2015, 119, $9402-$ 9410; b) X. Jiao, C. Lin, N. P. Young, C. Batchelor-McAuley, R. G. Compton, J. Phys. Chem. C 2016, 120, 13148-13158.

[29] E. Gileadi, M. A. Fullenwider, J. O. M. Bockris, J. Electrochem. Soc. 1966, 113, 926-930.

[30] P. Peljo, M. D. Scanlon, A. J. Olaya, L. Rivier, E. Smirnov, H. H. Girault, J. Phys. Chem. Lett. 2017, 8, 3564-3575.

Manuscript received: December 5, 2017

Revised manuscript received: January 13, 2018

Accepted manuscript online: January 28, 2018

Version of record online: February 22, 2018 